\title{
Relative Necessity and Propositional Quantification
}

\section{Alexander Roberts ${ }^{1}$}

Received: 23 January 2019 / Accepted: 30 October 2019/ Published online: 28 December 2019

(C) The Author(s) 2019

\begin{abstract}
Following Smiley's (The Journal of Symbolic Logic, 28, 113-134 1963) influential proposal, it has become standard practice to characterise notions of relative necessity in terms of simple strict conditionals. However, Humberstone (Reports on Mathematical Logic, 13, 33-42 1981) and others have highlighted various flaws with Smiley's now standard account of relative necessity. In their recent article, Hale and Leech (Journal of Philosophical Logic, 46, 1-26 2017) propose a novel account of relative necessity designed to overcome the problems facing the standard account. Nevertheless, the current article argues that Hale \& Leech's account suffers from its own defects, some of which Hale \& Leech are aware of but underplay. To supplement this criticism, the article offers an alternative account of relative necessity which overcomes these defects. This alternative account is developed in a quantified modal propositional logic and is shown model-theoretically to meet several desiderata of an account of relative necessity.
\end{abstract}

Keywords Relative necessity $\cdot$ Absolute necessity $\cdot$ Logical necessity $\cdot$ Modal logic

There are many different notions of necessity. For example, it may well be practically impossible for someone to run from London to Oxford in under three hours, but surely it is physically possible for that person to do so. Similarly, it is physically necessary that nothing goes faster than the speed of light, but many would claim that it is metaphysically possible for something to do so.

Although there are various different kinds of necessity, it is commonly assumed that there is an absolute notion of necessity which is broader than all other notions of necessity. Moreover, given this notion of absolute necessity, it is also widely assumed that all other kinds of necessity can be characterised in terms of it. Indeed, since Smiley's [15] influential proposal, it has become standard practice to characterise notions of relative necessity in terms of certain absolutely strict conditionals. According to

Alexander Roberts

alexander.roberts@univ.ox.ac.uk

1 University of Oxford, Oxford, UK 
this proposal, the relative necessity of some proposition $p$ can be characterised in terms of the absolute strict implication of $p$ by some suitable sentential constant. For instance, on this view, the physical necessity of a proposition $p$ is equated with the absolute strict implication of $p$ by a sentential constant expressing the laws of physics.

Nevertheless, Humberstone [11] and others have highlighted various flaws with Smiley's now standard account of relative necessity. Aware of these flaws, in their recent article Hale and Leech [10] propose a novel account of relative necessity designed to overcome the problems facing the standard account. However, although Hale \& Leech's account is an improvement on the standard account in certain respects, it suffers from its own defects. The current article outlines these defects, some of which Hale \& Leech are aware of but underplay, and emphasises their seriousness. The article then defends an account of relative necessity without these defects, to which the use of propositional quantification is key. It is then shown model-theoretically that this new account meets several desiderata of an account of relative necessity. The article concludes with a brief discussion of certain complexities which arise if various background assumptions are lifted.

\section{Absolute Necessity}

On the prevailing conception, for a notion of necessity to be absolute is for it to be the broadest necessity: that is, it is a matter of necessity that whatever is absolutely necessary is necessary in any other sense. This conception is found in both McFetridge [13] and Hale's [9] classic discussions of absolute necessity. Moreover, in recent work, Bacon [2] has shown how to formalise this conception in a typed quantified modal language with quantification into operator position.

A slightly less formal version of Bacon's official characterisation can be stated with the aid of the following notion: ${ }^{1}$

ALAB encodes the popular idea that one notion of necessity is as broad as another just in case, as a matter of necessity (in every sense), the latter deems as necessary

\footnotetext{
${ }^{1}$ Bacon's official working language is a functional type theory in which each type has a corresponding universal quantifier used to generalise universally over things of that type. In Bacon's [2] terms, ALAB can be written as follows:

$$
\square_{i} \geq \square_{j} \leftrightarrow \forall \square_{k}\left(N \square_{k} \rightarrow \forall p \square_{k}\left(\square_{i} p \rightarrow \square_{j} p\right)\right)
$$

Here, the subscripted boxes are expressions which take formulas as arguments and result in another sentence, ' $\geq$ ' is an expression which takes expressions of the same type as subscripted boxes as arguments and results in a sentence, and ' $N$ ' is a predicate which takes as arguments expressions of the same type as subscripted boxes to produce a sentence. On their intended interpretation, ' $\geq$ ' denotes the relation of beingas-broad-as and ' $N$ ' denotes the property (of properties of propositions) of being-a-necessity-operator, which is defined in Bacon [2, p. 735]. Although ALAB in the main body of text mixes object- and metalanguage, it should be understood as an informal statement of the above formalisation which does not. Indeed, similar principles in the main body of text may always be understood as informal statements of their formalisations in this typed formal language.
} 
every proposition which the former deems necessary; the broader a notion of necessity, the more stringent it is. Put alternatively, a notion of necessity is as broad as another just in case, as a matter of necessity (in every sense), any proposition which is possible according to the latter is possible according to the former; the broader a notion of necessity, the more inclusive its corresponding notion of possibility. To take an example, it is plausible that physical necessity is as broad as practical necessity since, as a matter of necessity (in every sense), anything which is practically possible is physically possible too. Given ALAB, the claim that absolute necessity ( $\square$ ) is the broadest necessity can be expressed as follows: ${ }^{2}$
Absolute $\forall p \square_{k}\left(\square p \rightarrow \square_{j} p\right)$ is true, whenever $\square_{k}$ and $\square_{j}$ are necessity operators

To reiterate, according to this characterisation, absolute necessity is as broad as any notion of necessity: for any proposition, it is a necessary matter (in every sense) that if that proposition is absolutely necessary it is necessary in every sense.

It bears emphasis that ALAB and Absolute function as intended only if propositional necessitism is assumed, here understood as the view that, in any sense of necessity, it is necessary which propositions there are. Were propositional necessitism rejected, the propositional quantifiers in ALAB and Absolute would not range over all absolutely possible propositions, depriving the principles of their intended generality. As is typical in the literature, propositional necessitism will be a working assumption in what follows.

According to one popular view, a natural candidate for absolute necessity is logical necessity. However, it is often unclear how logical necessity - thought of as a propositional operator, not a predicate of expressions - is intended to be characterised. Nevertheless, under the assumption of Booleanism, according to which propositions form a Boolean algebra under the usual truth-functional operations, logical necessity can be characterised quite naturally. In the Boolean setting, there is a unique tautologous proposition - the top element of the algebra — which may be thought of as the logical truth. Thus, in that setting, it is highly natural to characterise logical necessity as simply being identical with the tautologous proposition. ${ }^{3}$ Given such elegance, the article will simply adopt the hypothesis of Booleanism to provide a stable background to the discussion. To be more precise, the article will adopt the assumption that propositions form a complete Boolean algebra under the usual truth-functional operations, an assumption which since Fine [5] is familiar to the study of propositionally quantified modal logics. As will be seen, Booleanism is an elegant working setting in which to adjudicate competing accounts of relative necessity. Moreover, the final section discusses how the arguments of the article may be adapted to non-Boolean settings.

\footnotetext{
${ }^{2}$ The assumption that there is a unique broadest necessity will be justified shortly.

${ }^{3}$ Bacon [2] investigates this characterisation of logical necessity (although Bacon uses the term 'broad necessity' instead). See also Cresswell [3] and Suszko [17]. Dorr [4, p. 70] discusses how one might define a similar notion of necessity in non-Boolean settings.
} 
Interestingly, given only the austere characterisation of absolute necessity in Absolute, one should expect its logic to meet various conditions. To take a clear example, notice that Absolute strongly suggests that $\square$ will conform to the $\mathbf{4}$ axiom:

$4 \square p \rightarrow \square \square p$

After all, it is highly plausible that concatenations of modal operators express notions of necessity, and so $\square \square$ will itself be a species of necessity. Thus, given ALAB, $\square$ must be as broad as $\square \square$. Similarly, it is also plausible that $\square$ conforms to the $\mathbf{T}$ axiom, since surely $\square$ is as broad as some alethic notion of necessity:

$$
\mathbf{T} \square p \rightarrow p
$$

Finally, it is equally plausible that $\square$ conforms to the $\mathbf{K}$ axiom which is partly characteristic of normal modal operators:

$$
\mathbf{K} \square(p \rightarrow q) \rightarrow(\square p \rightarrow \square q)
$$

Given that it is natural to expect that the ultimate logic of $\square$ should be closed under modus ponens, necessitation, and uniform substitution, $\mathbf{S 4}$ will be treated as a lower bound on the logic of absolute necessity. ${ }^{4}$

As a final piece of set up, we make the working assumption that absolute necessity is unique. This assumption is usually taken to be implicit, however we shall enforce it explicitly by requiring notions of necessity to be individuated by a criterion of intensional equivalence. ${ }^{5}$

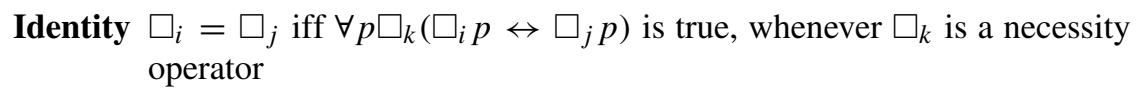

Informally, Identity states that notions of necessity are the same just in case it is necessary (in all senses) that they apply to exactly the same propositions. This has the consequence that absolute necessity is unique, for if $\square$ and $\square^{\prime}$ are absolute notions of necessity, then by Absolute they are as broad as one another. However, by ALAB and Identity it must be that $\square$ and $\square^{\prime}$ are one and the same.

\footnotetext{
${ }^{4}$ Indeed, Bacon [2] proves in his particular system of higher-order logic that absolute necessity meets all the principles of $\mathbf{S} \mathbf{4}$ including a rule of necessitation.

${ }^{5}$ Identity will be rejected by those who think that intensional equivalence (according to every notion of necessity) is too coarse-grained a criterion for individuating notions of necessity. According to that conception, there may be several absolute notions of necessity, all of which are as broad as all other notions of necessity. However, those who reject Identity may still wish to engage in the project of characterising non-absolute notions of necessity in terms of the absolute necessities. See both Dorr [4, sect. 5] and Bacon's [2] discussion of his 'modalized functionality' principle for related details.
} 


\section{Relative Necessities}

\subsection{Characterising Relative Necessities}

Relative necessities contrast with absolute necessity. Broadly speaking, relative necessities are types of parameterised modals. For example, one might think of physical necessity - a paradigm relative necessity — as necessity given the laws of physics. Similarly, one might think of metaphysical necessity as necessity given some particular body of metaphysical truths. In light of these glosses, it is tempting to think that in some sense all non-absolute, relative notions of necessity can be characterised in terms of an absolute notion of necessity. For a given notion of relative $i$-necessity, all that would be required is that one specifies 'necessity given the $i$-laws' purely in terms of absolute necessity, some vocabulary $\xi_{i}$ expressing the $i$-laws, and the logical symbols. More carefully, for each notion of relative necessity $\square_{i}$, there must be a true claim of the following form:

$\square\left(\square_{i} \phi \leftrightarrow \psi\right)$, where $\psi$ contains $\phi$ (and no propositional variables free in $\phi$ are bound in $\psi$ ), and $\phi$ otherwise may contain only $\square, \xi_{i}$, and logical symbols

In this sense, each relative necessity could characterised in terms of an absolute notion of necessity. Indeed, doing so is perhaps one way of making literal sense of the popular metaphorical claim that absolute necessity is the source of all necessity.

Before considering different characterisations of relative necessity, three points bear emphasis. First, it is helpful to clarify the target notion of relative necessity itself. Thus, to make the target explicit, the class of necessities which will be of interest to the current article are exactly those notions of necessity whose logic is characterised by a Kripke frame: the Kripke necessities. The target therefore does not include so-called 'linguistic necessities' like 'logical truth', which are expressed by predicates of sentences as opposed to propositional operators. Moreover, the target also excludes notions of necessity which are expressed by non-normal modal operators. Such non-normal notions of necessity may be rightfully classified as necessities in some broader sense; for example, Bacon's [2] notions of necessity are not required to meet the $\mathbf{K}$ axiom. However, it is reasonable not to target the non-normal necessities for current purposes: the Kripke necessities themselves constitute an extremely natural class of necessities which is highly non-trivial to characterise in terms of absolute necessity. Furthermore, the Kripke necessities include the paradigm relative necessities, such as physical and metaphysical necessity, which initially motivated the characterisation project. In Section 4.2.3, it will be proven that there is a precise sense in which the proposed definition of the relative necessities captures exactly the Kripke necessities.

Second, to simplify the complexity of the official object-language, it will contain $\square$ as its only necessity operator. The object-language will therefore not contain modal operators corresponding to each notion of relative necessity. Instead, relative necessities will be introduced as metalinguistic abbreviations for complex object-language formulae. Due to this simplification, the use of 'relative necessity operator' throughout should be understood as covering the object-language formulae corresponding to 
such metalinguistic abbreviations. ${ }^{6}$ However, to reiterate, when the article provides its positive account of relative necessities, it will be shown that there is a precise sense in which each target relative notion of necessity is expressed by some complex formulae of the object-language.

Finally, the use of ' $i$-laws' should be understood very liberally. The ' $i$-laws' for a given notion of relative $i$-necessity are just some body of propositions which serve to relativise the notion of necessity, and need not have the status of scientific laws. For example, in the case of necessity given Theresa May's goals in March 2019, the relevant body of propositions will comprise just those propositions which she wished to realise (for instance, that Parliament passes her Withdrawal Agreement). This liberal understanding of ' $i$-laws' is totally standard to the literature on relative necessities.

\subsection{Smiley's Standard Account}

Historically, the 'necessity given the $i$-laws' locution has been formalised in terms of simple absolutely strict conditionals. According to the standard account, for a proposition $p$ to be $i$-necessary is for $p$ to be absolutely strictly implied by some sentential constant which expresses the $i$-laws. The idea is widespread and at least dates back to Smiley's [15] influential discussion:

If we define $\mathbf{O} A\left[\square_{i} A\right]$ as $\mathbf{L}(T \rightarrow A)[\square(T \rightarrow A)]$ then to assert $\mathbf{O} A\left[\square_{i} A\right]$ is to assert that $T$ strictly implies $A$ or that $A$ is necessary relative to $T$. Since the pattern of the definition is independent of the particular interpretation that may be put on $T$ we can say that to the extent that the standard alethic modal systems embody the idea of absolute or logical necessity, the corresponding $\mathbf{O}$-systems embody the idea of relative necessity-necessity relative to an arbitrary proposition or body of propositions. They should therefore be appropriate for the formalisation of any modal notion that can be analysed in terms of relative necessity.

To take an example, according to Smiley's account a proposition $p$ is physically necessary iff $\square(L \rightarrow p)$ is true, where $L$ is a sentential constant expressing the laws of physics. ${ }^{7}$ Similarly, according to his account a proposition $p$ is metaphysically necessary iff $\square(M \rightarrow p)$ is true, where $M$ is some sentential constant expressing the 'laws' of metaphysics (such as claims of identity and distinctness, and various essentialist claims). Call this the standard account:

SA $\square_{i} \phi=_{d f} \square(\Psi \rightarrow \phi)$, where $\Psi$ is a sentential constant which expresses the ' $i$-laws'

\footnotetext{
${ }^{6}$ Similarly, 'necessity operator' should be understood as meaning 'relative necessity operator or the absolute necessity operator'.

${ }^{7}$ Williamson [19, pp. 462-463] notes that various truths of metaphysics must either be expressed by $L$ or conjoined to it for reasons of adequacy.
} 
Despite the influence of Smiley's standard account, Humberstone [11] and others have highlighted various flaws with it. ${ }^{8}$ Moreover, in their recent article, Bob Hale and Jessica Leech have carefully articulated and developed the problems facing the standard account. They raise two central issues. First, given that S4 is a lower bound on the logic of $\square$, every notion of relative necessity will conform to its own version of the 4 axiom. Second, for the same reason, any relative necessities $\square_{i}$ and $\square_{j}$ will conform to a bizarre 'mixed' 4 axiom $\square_{i} p \rightarrow \square_{j} \square_{i} p$. Although the former is a special case of the latter, each problem shall be considered separately in order to appreciate the nature of the issue.

To begin with the former, consider the following $\mathbf{S 4}$ valid schematic line of reasoning:

(1) $\square \phi \rightarrow(\psi \rightarrow \square \phi)$

PL Tautology

(2) $\square(\square \phi \rightarrow(\psi \rightarrow \square \phi))$

Necessitation, (1)

(3) $\square \square \phi \rightarrow \square(\psi \rightarrow \square \phi)$

$\mathbf{K}, \mathrm{MP},(2)$

(4) $\square \phi \rightarrow \square \square \phi$

(5) $\square \phi \rightarrow \square(\psi \rightarrow \square \phi)$

PL, (3), (4)

Now consider any proposition $p$ and sentential constant $L$ expressing the laws of physics. The following is an instance of (5):

$$
\square(L \rightarrow p) \rightarrow \square(L \rightarrow \square(L \rightarrow p))
$$

Yet, by $\mathbf{S A}$, this just abbreviates an instance of the $\mathbf{4}$ axiom for physical necessity. And the argument generalises to every notion of relative necessity. But surely not all notions of relative necessity in the target sense conform to a version of 4 . To take an example, consider the notion of practical necessity, of what is necessary given our current practical means. At some point in recent history, it was practically impossible for us to engineer smartphones. However, at that point, surely it was within our practical means to engineer technology which would provide us with the practical means to engineer smartphones. Thus, although it was practically impossible for us to engineer smartphones, it was still practically possibly possible for us to do so, which constitutes a failure of $\mathbf{4}$ for practical necessity.

To see the second problem, consider the sentential constant $L$ expressing the actual laws of physics and the sentential constant $M$ expressing the 'laws' of metaphysics. The following is another instance of (5):

$$
\square(L \rightarrow p) \rightarrow \square(M \rightarrow \square(L \rightarrow p))
$$

\footnotetext{
${ }^{8}$ Humberstone [11] attributes the problems for Smiley's account to an observation made by Kit Fine in conversation.
} 
Yet, by SA, this just abbreviates the claim that if a given proposition $p$ is physically necessary then it is metaphysically necessary that $p$ is physically necessary. But for some propositions this is false: even though superluminal travel is physically impossible, there are metaphysically possible worlds with different laws of physics from which it is physically possible that bodies go faster than the actual speed of light.

Given that the target notions of relative necessity are all the Kripke necessities, it is simply a failure of SA that it is only able to characterise those Kripke necessities whose logic includes the $\mathbf{4}$ axiom for the necessity operator in question. The issue is that the standard account attempts to formalise the idea that $i$-necessity is absolute necessity relative to the $i$-laws with the use of sentential constants which express the $i$-laws of a specific world. However, this use of constants generates the $\mathbf{4}$ axiom and mixed 4 axiom issues. What is needed is some means of formalising the variability of the $i$-laws from absolutely possible world to world in the language of propositional modal logic.

\section{Hale \& Leech's Proposal}

\subsection{The Proposal}

Hale \& Leech clearly appreciate the force of the above concerns. After highlighting the shortcomings of Humberstone's $[11,12]$ two-dimensional solution, they offer their own definition of relative necessity. According to Hale \& Leech's proposal, each relative necessity operator $\square_{i}$ is to be defined in terms of a corresponding propositional operator $\pi_{i}$. In the case of, say, physical necessity, here denoted as $\square_{\text {phys }}$, the claim $\pi_{p h y s}(p)$ expresses that $p$ is a law of physics.

For their first attempt, Hale $\&$ Leech propose that $i$-necessity can be defined as follows (where $q$ is the first variable not free in $\phi$ under some fixed ordering) $:^{9}$

$$
\text { HL1 } \square_{i} \phi={ }_{d f} \exists q\left(\pi_{i}(q) \wedge \square(q \rightarrow \phi)\right)
$$

In other words, $p$ is $i$-necessary iff there is some $i$-law which strictly implies $p$. An instance of HL1 is the claim that $p$ is physically necessary iff there is some law of physics which strictly implies it.

One might immediately notice that this is somewhat weak. After all, surely the physical necessities ought to be closed under conjunction. Indeed, all instances of the following schema are valid in any normal modal logic:

$$
\mathbf{K} \wedge\left(\square_{i} \phi \wedge \square_{i} \psi\right) \rightarrow \square_{i}(\phi \wedge \psi)
$$

\footnotetext{
${ }^{9}$ Hale and Leech [10, p. 13] do not state their account schematically, but instead state it in terms of propositional variables. When stated in schematic form, one needs the condition that $q$ is the first variable not free in $\phi$ in order to avoid clash of variables.
} 
But HL1 does not guarantee closure under conjunction, and so would generate nonvalid instances of $\mathbf{K} \wedge .{ }^{10}$ To see this informally, imagine there were only two laws $r$ and $r^{\prime}$ such that $r$ strictly implies $p$ and $r^{\prime}$ strictly implies $q$. Since neither $r$ nor $r^{\prime}$ strictly implies $p \wedge q, \mathbf{K} \wedge$ fails for physical necessity.

Hale \& Leech are aware of this issue, and offer a more sophisticated definition of relative necessity to handle the problem. In this definition, $\Gamma$ is a finite set of propositional variables $q_{1}, \ldots, q_{n}$, the first $n$ variables (given some fixed order) not free in $\phi$, and $\pi_{i}\left(q_{j}\right)$ abbreviates the finitary conjunction $\pi_{i}\left(q_{1}\right) \wedge \ldots \wedge \pi_{i}\left(q_{n}\right)$ :

$$
\text { HL2 } \square_{i} \phi=d_{f} \exists q_{1} \ldots \exists q_{n}\left(\pi_{i}\left(q_{j}\right) \wedge \square\left(\bigwedge_{q_{j} \in \Gamma} q_{j} \rightarrow \phi\right)\right)
$$

According to this account, $p$ is $i$-necessary iff there are some propositions, the $i$ laws, the conjunction of which strictly implies $p$. An instance of HL2 is the claim that $p$ is physically necessary iff there are some propositions, the laws of physics, the conjunction of which strictly implies $p$. As can be easily verified, HL2 validates $\mathbf{K} \wedge$ for each relative necessity.

In HL2, Hale \& Leech (p. 18, f.n. 23) ultimately want to treat $n$ as variable. They recognise that this requires a departure from the resources of ordinary quantified modal propositional logic. For instance, they entertain the idea of introducing explicit quantification over the natural numbers into the object-language in order to permit variability. However, a more standard alternative would be to state their ultimate account of relative necessity in terms of a countably infinite disjunction of finite HL2 instances. Relatedly, Hale \& Leech assume that $n$ is finite. In principle, this finitary simplification may ultimately have to be lifted in order to accommodate relative necessities based on infinitely many 'laws'. After all, it is surely absolutely possible for there to be such notions of relative necessity. For example, it is absolutely possible that Theresa May had infinitely many different goals in March 2019, in which case an infinite body of propositions would restrict necessity given Theresa May's goals in March 2019. As a result, the ultimate regimentation of HL2 may have to be stated in a language with infinitary conjunction operator which binds arbitrary sets of formulas. Although this creates additional technical complexity, there should be no technical obstacle to introducing such infinitary resources to modal languages. Indeed, some key invariance results in modal logic only hold in modal languages with infinite conjunction binding arbitrary sets of formulae. ${ }^{11}$ Nevertheless, the focus of

\footnotetext{
${ }^{10}$ Officially, Hale \& Leech do not provide a model-theoretic semantics or definition of validity for their language, although they (p. 15) note that "the most obvious method" would be to follow Fine's classic [5] semantics. In Fine's framework, propositions are interpreted as sets of worlds, propositional variables and quantifiers range over the power set of worlds in a model, and validity in a model is defined as usual. Hale \& Leech (p. 16) emphasise that they are "reasonably confident that with further work, [their] informal [proof] sketches can turned into rigorous model-theoretic [proofs]". At points, their informal presentation will be followed when discussing their view; however, the current paper's positive proposal will be developed explicitly in the setting of a model-theoretic framework similar to that of Fine [5]. Given that similarity, all claims of validity regarding Hale \& Leech's proposal should be understood in terms of the notion of validity defined in Def. 5 below.

${ }^{11}$ See Goranko and Otto [8, pp. 270-273]; see also van Benthem [1, pp. 30-31] for an accessible explanation of the invariance results.
} 
what follows will be on the simple HL2 account, since it shares many features of interest with these more sophisticated refinements.

Neatly, HL2 overcomes the two above problems with $\mathbf{S 4}$. To see this, consider the 4 axiom for $\square_{i}$ according to HL2:

$$
\begin{gathered}
4^{\prime} \exists q_{1} \ldots \exists q_{n}\left(\pi_{i}\left(q_{j}\right) \wedge \square\left(\bigwedge_{q_{j} \in \Gamma} q_{j} \rightarrow p\right)\right) \rightarrow \exists q_{1} \ldots \exists q_{n}\left(\pi_{i}\left(q_{j}\right)\right. \\
\left.\wedge \square\left(\bigwedge_{q_{j} \in \Gamma} q_{j} \rightarrow \exists q_{1} \ldots \exists q_{n}\left(\pi_{i}\left(q_{j}\right) \wedge \square\left(\bigwedge_{q_{j} \in \Gamma} q_{j} \rightarrow p\right)\right)\right)\right)
\end{gathered}
$$

This formula is not a theorem of S4 (or even S5): it is invalidated in models similar to the one given in Section 4.2.1 below. Thus, the definition of relative necessity does not force all notions of relative necessity to conform to the $\mathbf{4}$ axiom. A similar argument shows that HL2 overcomes the 'mixed' $\mathbf{4}$ problem too.

To close the discussion of HL2, it may be assumed on behalf of Hale \& Leech that a given notion of relative necessity $\square_{i}$ has a $d u a l$ notion of possibility $\diamond_{i}$ to be understood as follows (where again $q_{1}, \ldots, q_{n}$ are the first $n$ variables, given some fixed order, not free in $\phi)$ :

$$
\text { HL2 } \diamond \diamond_{i} \phi=d_{f} \neg \exists q_{1} \ldots \exists q_{n}\left(\pi_{i}\left(q_{j}\right) \wedge \square\left(\bigwedge_{q_{j} \in \Gamma} q_{j} \rightarrow \neg \phi\right)\right)
$$

Thus, in logically equivalent form, the formula $\diamond_{i} p$ abbreviates the following formula:

$$
\forall q_{1} \ldots \forall q_{n}\left(\pi_{i}\left(q_{j}\right) \rightarrow \diamond\left(\bigwedge_{q_{j} \in \Gamma} q_{j} \wedge \phi\right)\right)
$$

That is to say, $p$ is $i$-possible according to HL2 iff for all $i$-laws it is absolutely possible that they are true along with $p$. If one endorses HL2, HL2 $\diamond$ is an extremely natural way to define relative notions of possibility; in what follows, talk of relative possibility on Hale \& Leech's view should be understood in these terms.

\subsection{Against HL2}

Despite the advantages of HL2 over the standard account, it suffers from several serious problems. The first is perhaps the most serious: HL2 fails to have the consequence that logical necessity is as broad as any relative necessity. Since logical necessity was considered as absolute because it was as broad as every notion of necessity, this is a seriously worrying defect. Indeed, put alternatively, Hale and Leech cannot even recognise a notion of necessity which is absolute in the required sense, which is arguably a fatal consequence of an account of relative necessity.

To see how this issue arises, fix on a given merely relative necessity, say physical necessity ( $\left.\square_{\text {phys }}\right)$, and consider the following consequence of Absolute:

$$
\text { [1] } \square(\square p \rightarrow \square \text { phys } p)
$$


Since $\square$ conforms to $\mathbf{T}$, we may conclude the following:

$$
\text { [2] } \square p \rightarrow \square \text { phys } p
$$

But one can see informally why Hale \& Leech's proposal does not validate [2]. Let $\top$ be the tautology $\forall p(p \rightarrow p)$. Given the usual definition of validity as truth at all worlds in all models, $T$ will be true at all worlds in every model and therefore valid. Since $\square$ has a normal modal logic, $\square T$ will be valid too. But consider some world $w$ in some model where there are no physical laws. Straightforwardly, at $w$ the following would be false:

$$
\text { [3] } \exists q_{1} \ldots \exists q_{n}\left(\pi_{i}\left(q_{j}\right) \wedge \square\left(\bigwedge_{q_{j} \in \Gamma} q_{j} \rightarrow \top\right)\right)
$$

The reason is that there are no physical laws at $w$, and so no proposition will be in the extension of $\pi_{i}$ there. But, by $\mathbf{H L 2}$, [3] is equivalent to $\square_{\text {phys }} \top$. Thus, [2] is not valid according to HL2, and so Absolute will not be valid either. Put alternatively, if there are $i$-lawless worlds for a given notion of $i$-necessity, absolute necessity will not be as broad as that notion of necessity.

Overall, it is worrying even if there is a single notion of relative necessity which absolute necessity is not as broad as. However, according to HL2 this phenomenon is quite widespread. After all, for many notions of relative $i$-necessity it will be absolutely possible that there are $i$-lawless worlds. For example, consider again necessity given Theresa May's goals in March 2019. Although May has various actual goals, it is absolutely possible that her goals could have been different. Instead of aiming for Parliament to pass her Withdrawal Agreement she might have desired for the United Kingdom to retain full membership of the European Single Market. Likewise, there is an absolute possibility in which May has no goals whatsoever, which would be a lawless world in the relevant sense. Since it is plausible that the phenomenon of absolutely possible $i$-lawless worlds arises for many notions of $i$-necessity, HL2 predicts that absolute necessity is not as broad as myriad relative necessities.

Hale \& Leech are aware of a version of the problem with Absolute but they attempt to underplay it. ${ }^{12}$ They write (pp. 18-19; their emphasis):

That [Absolute] should be validated is, no doubt, just what one would think, if one thinks about relative necessity in essentially world-terms: that is, so that what is physically necessary, for instance, is essentially just what is true throughout a restricted range of all logically possible worlds. For then, since anything logically necessary (i.e. true throughout the whole unrestricted range) must be true throughout any restriction of it, it must also be physically necessary. Our answer to this is that it is just a mistake to think of forms of relative necessity as fundamentally to be understood in world terms. If we drop that prejudice, then it can seem entirely

\footnotetext{
${ }^{12}$ They attribute the version of the problem they discuss to David Makinson; in their discussion, the problem is presented under a guise which is much different to the current one.
} 
natural and correct to characterise or define a form of relative necessity in such a way that logical necessity does not automatically ensure relative necessity.

Nonetheless, despite Hale \& Leech's insistence that Absolute should only be appealing to those in the grip of a worlds-based reduction of modality, it ought to be clear from the initial set up that this is just not true. The reason why Absolute is so plausible is because absolute necessity is characterised by the fact that it is as broad as all other notions of necessity. Indeed, how else would it be characterised? Its absoluteness consists in this feature. Moreover, claims of comparative broadness can be used to provide insight into the relations between different notions of necessity, which is surely an ambition of our modal theorising. ${ }^{13}$ The capacity to express such claims does not seem like an artefact of the worlds-based conception, but rather a requirement of modal theory.

At the heart of the above problem is Hale \& Leech's treatment of relative $i$ necessities at worlds at which there are no $i$-laws. According to HL2, at worlds with no $i$-laws no proposition is $i$-necessary since claims of $i$-necessity involve existential quantification over $i$-laws. But this is difficult to square with familiar glosses of relative necessities. For example, consider the following typical necessary condition on physical possibility:

PP If a proposition is physically possible at world, it is logically consistent with the world's laws of physics.

Indeed, Hale \& Leech (p. 2) appear to endorse this very condition:

Thus on one common view, physical necessity is a matter of following from the laws of physics, and physical possibility is compatibility [i.e. logical consistency] with them.

However, according to HL2/HL2 $\diamond$, at worlds with no laws of physics it is vacuously true that every proposition whatsoever (including contradictory propositions) is physically possible. Yet this violates the highly plausible condition that, whatever the circumstances, only logically possible propositions are physically possible. Moreover, as stressed above, since no proposition is physically necessary at lawless worlds according to HL2, not even arbitrary tautologies will be physically necessary there either. But on the typical definition of validity as truth at all worlds in all models, it would follow that notions of relative necessity do not even have a normal modal logic, since they would fail to conform to the rule of necessitation.

There are thus several concerns facing HL2. There are the minor issues that HL2 is too simple to handle cases in which there are infinitely many $i$-laws, and that

\footnotetext{
${ }^{13}$ For recent examples of such insight, see Williamson [19] and Bacon [2]. See also Hale's [9] classic and influential discussion of absolute necessity.
} 
HL2 must be modified to permit variability in the number of laws. However, more worryingly, there are the major issues regarding the failure of Absolute, and the mishandling of lawless worlds, the latter of which is essentially responsible the former. Finally, there is the subsidiary problem of the rule of necessitation failing for notions of relative necessity, which again is an artefact of the mishandling of lawless worlds. Together, these problems motivate the need for a new definition of relative necessity, preferably in terms of absolute necessity.

Nevertheless, HL2 is clearly on the right track, as witnessed by its predictions in cases with a non-zero finite number of $i$-laws. As a consequence, it might seem that a slightly generalised version of HL2 fares much better. To see this, imagine that the object-language is enriched with an infinitary conjunction operator which binds arbitrary sets of formulas and equates empty conjunctions with the tautologous proposition. In this infinitary setting, HL2 can be generalised to the claim that for a proposition to be $i$-necessary is for it to be absolutely strictly implied by the arbitrary conjunction of the $i$-laws. Without formalising the proposal, one would expect this generalised version of HL2 to avoid most of the problems which faced HL2. For instance, clearly the generalised version would no longer incorporate the restriction to finitely many laws. Moreover, at $i$-lawless worlds, the restricting proposition for relative $i$-necessity would simply be the tautologous proposition. Consequently, at such worlds relative $i$-necessity collapses into absolute necessity, thus ensuring Absolute. Indeed, Strohminger and Yli-Vakkuri [16] sketch an analysis of relative necessity similar to HL2 in terms of infinite conjunction of exactly this kind, and suggest that they expect it to overcome the problems facing HL2. Arguably, the only remaining issues are that the account requires it to be absolutely necessary that the $i$-laws form a set, but this issue could perhaps be finessed with even more extreme infinitary resources.

However, rather than complicate the propositional language by resorting to any infinitary measures whatsoever, there is a much simpler option available. Put intuitively, the idea is to exploit the fact that, given our particular assumption of Booleanism, the arbitrary conjunction of the $i$-laws will just be the weakest proposition that strictly implies all $i$-laws. Yet, as will be shown, that proposition can be characterised simply in terms of propositional quantification and the ' $i$-law' predicate. Thus, the relative $i$-necessity of a given proposition can be characterised as that proposition's being strictly implied by the weakest proposition that strictly implies all $i$-laws, which is statable without appeal to any infinitary resources. ${ }^{14}$ The next section develops this simple idea carefully and shows that it overcomes all of the problems facing $\mathbf{H L} \mathbf{2}$ in addition to the initial $\mathbf{S 4}$ problems.

\footnotetext{
${ }^{14}$ Indeed, Strohminger and Yli-Vakkuri [16, p. 6, n. 11; p. 7, n. 12] themselves suggest that a 'higherorder analysis' of relative necessity is needed, which the following simpler proposal may be viewed as providing.
} 


\section{A New Proposal}

\subsection{Regimentation}

The aim of this section is to develop the above characterisation carefully. On the proposed way of developing this characterisation, Absolute will be validated and the issue with lawless worlds will be rectified. Moreover, the proposal will ensure a normal modal logic for each notion of relative necessity. Furthermore, due to the similarities of the new proposal with HL2, the new proposal will also overcome the S4-related problems that afflicted the standard account. Indeed, more generally, the new proposal will yield the welcome result that its notions of relative necessity are exactly the Kripke necessities, thus ensuring that it captures the initial target notion of relative necessity.

The exact statement of the new proposal shall be postponed until some technical resources have been introduced. The technical resources are to be introduced modeltheoretically, so we must first specify a language.

Def. 1 The language $\mathscr{L}$ is defined using: countably many propositional variables $(p, q, \ldots)$; countably many monadic predicate constants $\left(\pi_{1}, \pi_{2}, \ldots\right)$; conjunction $(\wedge)$ and negation $(\neg)$ as the only truth-functional connectives; the unary modal operator $\square$ (absolute necessity), and a universal quantifier binding propositional variables $(\forall)$.

The well-formed formulas of the language are given by the following rule (where $\alpha$ is a propositional variable and $\xi$ is a monadic predicate constant):

$$
\phi:=\alpha|\xi(\alpha)| \neg \psi|(\psi \wedge \chi)| \forall \alpha(\psi) \mid \square \psi
$$

We write $\exists$ as an abbreviation for the string $\neg \forall \neg$ and $\diamond$ as an abbreviation for the string $\neg \square \neg$. We introduce $\rightarrow, \vee$, and $\leftrightarrow$ by the usual abbreviations.

The class of models for this language shall not permit contingency in what propositions there are. Thus, to this end, we shall use constant domain models for quantified propositional logics; the class of models is similar to that defined in the classic Fine [5] which, in effect, employs a constant domain model theory.

Def. 2 A model $\mathfrak{M}$ of the language is a pair $\langle\mathscr{W}, \mathscr{V}\rangle$, where:

$\mathscr{W}$ is a non-empty set ('the worlds'), and

$\mathscr{V}$ is a total function ('the interpretation function') from pairs $\langle\xi, w\rangle$ (for $\xi$ a predicate constant, and $w \in \mathscr{W})$ to subsets of $\mathscr{P}(\mathscr{W})$.

Def. 3 A variable assignment $g$ for the language is a total function from propositional variables to subsets of $\mathscr{W}$. We write $g(\alpha / d)$ for a variable assignment which differs from $g$ at most in that it assigns $d$ to $\alpha$. 
The next step is to define a truth at a world on an assignment.

Def. 4 We define ' $(\mathfrak{M}, w) \models_{g} \phi$ ' ('true at $w$ on $g$ ') for $\phi$ a well-formed formula, $\mathfrak{M}=\langle\mathscr{W}, \mathscr{V}\rangle$, and $w \in \mathscr{W}$ recursively in the following manner. In this definition, $\alpha$ is propositional variable, $\xi$ is a monadic predicate constant, and $\phi$ and $\psi$ are both well-formed formulae.

$$
\begin{aligned}
& (\mathfrak{M}, w) \models_{g} \alpha \text { iff } w \in g(\alpha) \\
& (\mathfrak{M}, w) \models_{g} \xi(\alpha) \text { iff } g(\alpha) \in \mathscr{V}(\xi, w) \\
& (\mathfrak{M}, w) \models_{g} \neg \phi \text { iff }(\mathfrak{M}, w) \models_{g} \phi \\
& (\mathfrak{M}, w) \models_{g} \phi \wedge \psi \text { iff }(\mathfrak{M}, w) \models_{g} \phi \text { and }(\mathfrak{M}, w) \models_{g} \psi \\
& (\mathfrak{M}, w) \models_{g} \square \phi \text { iff }(\mathfrak{M}, u) \models_{g} \phi \text {, for all } u \in W \\
& (\mathfrak{M}, w) \models_{g} \forall \alpha(\phi) \text { iff }(\mathfrak{M}, w) \models_{g(\alpha / p)} \phi \text {, for all } p \in \mathscr{P}(W)
\end{aligned}
$$

Def. 5 A formula $\phi$ is valid in $\mathfrak{M}=\langle\mathscr{W}, \mathscr{V}\rangle$ iff $(\mathfrak{M}, w) \models_{g} \phi$ for all $w \in \mathscr{W}$, and all variable assignments $g$. A formula $\phi$ is valid iff it is valid in all models.

Note that this semantics validates the Barcan formula and its converse for the propositional quantifier, as is standard in necessitist logics which validate S5. ${ }^{15}$

With this technical apparatus, we are now in a position to state the new proposed definition of relative necessity. Intuitively, the idea is that $p$ is $i$-necessary at a world iff it is strictly implied by the weakest proposition that strictly implies every $i$-law at that world. For example, $p$ is physically necessary at a world iff it is strictly implied by the weakest proposition that strictly implies every physical law at that world. To appreciate this idea, it may help to emphasise that in cases where there are finitely many $i$-laws at a given world, the weakest proposition that strictly implies every $i$ law at that world will just be the proposition expressed by the finite conjunction of the $i$-laws. As a result of this, the new account of relative necessity will agree with the welcome predictions of $\mathbf{H L 2}$ in cases of a non-zero finite number of $i$-laws. To state the definition formally, we introduce two metalinguistic abbreviations:

$$
\begin{aligned}
& \mathscr{O}^{\pi_{i}} p=d_{d f} \quad \forall q\left(\pi_{i}(q) \rightarrow \square(p \rightarrow q)\right) \\
& \mathscr{L}^{\pi_{i}} p={ }_{d f} \quad \mathscr{O}^{\pi_{i}}(p) \wedge \forall q\left(\mathscr{O}^{\pi_{i}}(q) \rightarrow \square(q \rightarrow p)\right)
\end{aligned}
$$

\footnotetext{
${ }^{15}$ The opening section noted that $\mathbf{S 4}$ is a lower bound on the logic of absolute necessity; indeed, this is a theorem of Bacon's [2] characterisation of absolute necessity. To argue that $\mathbf{S 5}$ is an upper bound on the logic of absolute necessity, Bacon adapts an informal argument due to Williamson [18, pp. 114] which appeals to Scroggs's [14] theorem that every proper extension of S5 is the logic of a single finite frame. The current model theory validates $\mathbf{S 5}$ for absolute necessity as a simplifying working hypothesis.
} 
Put informally, $\mathscr{O}_{i}^{\pi} p$ means that $p$ strictly implies all propositions that are $i$-laws, and $\mathscr{L}^{\pi_{i}} p$ means that $p$ is the weakest such proposition with respect to strict implication. To aid one's understanding of these notions, it may help to notice that since propositions are modelled as sets of absolutely possible worlds, one proposition's strictly implying another is modelled as the former being a subset of the latter. Given this equivalence, $\mathscr{O}^{\pi_{i}} p$ can be understood as the claim that $p$ is a subset of all $i$ laws, and $\mathscr{L}^{\pi_{i}} p$ can be understood as the claim that $p$ is the greatest lower bound on the $i$-laws with respect to subset inclusion. When $\mathscr{L}^{\pi_{i}} p$ is true at a world on a given assignment, whatever $p$ is assigned will be referred to as the $i$-lawful proposition of that world. Informally, one might wish to think of the $i$-lawful proposition as the arbitrary conjunction the $i$-laws, where the empty conjunction is simply the tautologous proposition. However, an advantage of the $\mathscr{L}^{\pi_{i}}$ characterisation is that it defines the $i$-lawful proposition simply in terms of already available propositional quantification.

It is important to stress that since propositions are treated as sets of worlds in a given model, the domain of propositions (i.e. the power set of the worlds in the model) will form a complete Boolean algebra under the usual set-theoretic operations in the sense that every subset of propositions has a least upper bound and a greatest lower bound with respect to subset inclusion. Thus, for any set of propositions there is guaranteed to be a weakest proposition that strictly implies all its members. Helpfully, this means that at each world exactly one proposition will satisfy $\mathscr{L}^{\pi_{i}}$ for a given predicate $\pi_{i}$.

The new definition of relative necessity can now be stated as follows (where $q$ is the first variable not free in $\phi$ ):

$$
\mathbf{R N} \square_{i} \phi={ }_{d f} \exists q\left(\mathscr{L}^{\pi_{i}}(q) \wedge \square(q \rightarrow \phi)\right)
$$

To restate the intuitive gloss: $i$-necessity at a world is a matter of being strictly implied by the weakest proposition that strictly implies all $i$-laws at that world.

\subsection{Results}

In certain respects, RN is similar to both HL2 and SA. This section will highlight these similarities, but also emphasise its crucial differences. These differences will ensure that RN overcomes each of the problems which HL2 and SA faced.

\subsubsection{Axiom Schema}

Recall that one problem with SA is that it validated all instances of the $\mathbf{4}$ axiom schema for every notion of relative necessity. It may be shown that $\mathbf{R N}$ does not have this feature.

Consider a relative necessity operator $\square_{i}$. According to $\mathbf{R N}$, the $\mathbf{4}$ axiom schema for $\square_{i}$ is stated as follows:

$$
\mathbf{4}_{i} \exists q\left(\mathscr{L}^{\pi_{i}}(q) \wedge \square(q \rightarrow \phi)\right) \rightarrow\left[\exists q \left(\mathscr{L}^{\pi_{i}}(q) \wedge \square\left(q \rightarrow \exists q\left(\mathscr{L}^{\pi_{i}}(q) \wedge \square(q \rightarrow \phi)\right)\right]\right.\right.
$$


The following is a countermodel to an instance of $\mathbf{4}_{i}$. Let $\mathfrak{M}=\langle\mathscr{W}, \mathscr{V}\rangle$, such that:

$$
\begin{aligned}
& \mathscr{W}=\{0,1,2\} \\
& \mathscr{V}\left(\pi_{i}, 0\right)=\{\{0,1\},\{0,1,2\}\} \\
& \mathscr{V}\left(\pi_{i}, 1\right)=\{\{0,1,2\}\} \\
& \mathscr{V}(\xi, w)=\emptyset, \text { for all } w \in \mathscr{W} \text { and all } \xi \text { otherwise }
\end{aligned}
$$

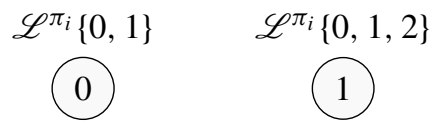

Let $g$ be a variable assignment such that $g(p)=\{0,1\}$. Say that $\mathbf{4}_{i}(p)$ is the instance of $\mathbf{4}_{i}$ with $p$ substituted for $\phi$. With $g$ as above, it can be checked that $(\mathfrak{M}, 0) \not \nvdash_{g}$ $\mathbf{4}_{i}(p)$.

The $\mathbf{4}_{i}$ axiom schema according to $\mathbf{R N}$ is a special case of the 'mixed' $\mathbf{4}_{i j}$ schema according to $\mathbf{R N}$, which is stated as follows:

$$
\mathbf{4}_{i j} \exists q\left(\mathscr{L}^{\pi_{i}}(q) \wedge \square(q \rightarrow \phi)\right) \rightarrow\left[\exists q \left(\mathscr{L}^{\pi_{i}}(q) \wedge \square\left(q \rightarrow \exists q\left(\mathscr{L}^{\pi_{j}}(q) \wedge \square(q \rightarrow \phi)\right)\right]\right.\right.
$$

As a consequence, instances of $\mathbf{4}_{i j}$ are invalidated by the model theory too.

\subsubsection{Absolute}

Next, it can be shown that $\forall p \square_{k}\left(\square p \rightarrow \square_{j} p\right)$ is valid whenever $\square_{k}$ and $\square_{j}$ are necessity operators. To this end, it is helpful to first establish that in a given model each notion of relative necessity corresponds to an accessibility relation in that model. To express this carefully, first say that for a set $S$ and $X$ a set of sets, the intersection of $X$ with respect to $S\left(\cap_{S} X\right)$ is defined as follows.

$$
\cap_{S} X=\{y \in S: y \in x \text { for all } x \in X\}
$$

One can then define the accessibility relation which corresponds to a given notion of relative necessity as follows.

Def. 6 For a predicate constant $\pi_{i}$ and model $\mathfrak{M}=\langle\mathscr{W}, \mathscr{V}\rangle, \mathscr{R}_{i}=\{\langle x, y\rangle: y \in$ $\left.\cap \mathscr{W}^{\mathscr{V}}\left(\pi_{i}, x\right)\right\}$

With this definition in mind, it is first helpful to observe that from a given world $w$ the worlds in which $w$ 's $i$-lawful proposition is true are exactly those worlds in the intersection of the extension of $\pi_{i}$ at $w$ with respect to the background space of worlds.

Theorem 1. For a model $\mathfrak{M}=\langle\mathscr{W}, \mathscr{V}\rangle$ and a variable assignment $g$, $(\mathfrak{M}, w) \models_{g}$ $\mathscr{L}^{\pi_{i}}(p)$ iff $\cap_{\mathscr{W}} \mathscr{V}\left(\pi_{i}, w\right)=g(p)$. 
Proof sketch. As mentioned previously, $(\mathfrak{M}, w) \models_{g} \mathscr{L}^{\pi_{i}}(p)$ equates to $g(p)$ being the greatest lower bound on $\mathscr{V}\left(\pi_{i}, w\right)$ with respect to $\subseteq$ in $\mathscr{P}(\mathscr{W})$ (hereafter these qualifications are left implicit). It thus suffices to show that $\cap \mathscr{W} \mathscr{V}\left(\pi_{i}, w\right)$ is also the greatest lower bound on $\mathscr{V}\left(\pi_{i}, w\right)$. It helps to separate the cases, so first assume that $\mathscr{V}\left(\pi_{i}, w\right) \neq \emptyset$. Clearly, for any $s \in \mathscr{V}\left(\pi_{i}, w\right), \cap \mathscr{W} \mathscr{V}\left(\pi_{i}, w\right) \subseteq s$. Moreover, $\cap \mathscr{W} \mathscr{V}\left(\pi_{i}, w\right)$ contains every $u$ such that $u \in s$ for all $s \in \mathscr{V}\left(\pi_{i}, w\right)$, so there is no lower bound $\Delta$ on $\mathscr{V}\left(\pi_{i}, w\right)$ such that $\cap \mathscr{W}^{\mathscr{V}}\left(\pi_{i}, w\right) \subset \Delta$. However, if $\mathscr{V}\left(\pi_{i}, w\right)=\emptyset$ then it is vacuously true that $\cap \mathscr{W} \mathscr{V}\left(\pi_{i}, w\right)$ is a lower bound on $\mathscr{V}\left(\pi_{i}, w\right)$. Moreover, given that $\mathscr{V}\left(\pi_{i}, w\right)=\emptyset$, it follows that $\cap \mathscr{W} \mathscr{V}\left(\pi_{i}, w\right)=\mathscr{W}$ and is thus the greatest lower bound on $\mathscr{V}\left(\pi_{i}, w\right)$.

Theorem 1 may then be employed to derive a semantic clause for a given notion of $i$-necessity using $\mathscr{R}_{i}$.

Theorem 2. (Accessibility) For a relative necessity $\square_{i}$, one may derive the following semantic clause: $(\mathfrak{M}, w) \models_{g} \square_{i} \phi$ iff for all $u$ such that $w \mathscr{R}_{i} u$, $(\mathfrak{M}, u) \models_{g} \phi$.

Proof. All steps are given by either the semantic clauses, Def. 6, or Theorem 1.

$$
\begin{aligned}
& (\mathfrak{M}, w) \models_{g} \exists q\left(\mathscr{L}^{\pi_{i}}(q) \wedge \square(q \rightarrow \phi)\right) \\
& \text { iff }(\mathfrak{M}, w) \models_{g(q / p)} \mathscr{L}^{\pi_{i}}(q) \wedge \square(q \rightarrow \phi) \text { for some } p \in \mathscr{P}(\mathscr{W}) \\
& \text { iff } \cap_{\mathscr{W}} \mathscr{V}\left(\pi_{i}, w\right)=g_{(q / p)}(q) \text { and }(\mathfrak{M}, w) \models_{g(q / p)} \square(q \rightarrow \phi) \\
& \text { iff }(\mathfrak{M}, u) \models_{g} \phi \text { for all } u \in \cap_{\mathscr{W}} \mathscr{V}\left(\pi_{i}, w\right)=g_{(q / p)}(q) \\
& \text { iff }(\mathfrak{M}, u) \models_{g} \phi \text { for all } u \text { such that } v \mathscr{R}_{i} u \text {. }
\end{aligned}
$$

To return to the main thread, notice that for each $\pi_{i}$ and model $\mathfrak{M}=\langle\mathscr{W}, \mathscr{V}\rangle, \mathscr{R}_{i} \subseteq$ $\mathscr{W} \times \mathscr{W}$. Thus, Theorem 2 can be used to establish that $\square p \rightarrow \square_{i} p$ is valid for $\square_{i}$ whenever $\square_{i}$ is a relative necessity operator. However, as can easily be checked by using the semantics and definition of validity, $\square$ obeys the rule of necessitation.

Necessitation If $\phi$ is valid, then $\square \phi$ is valid.

Given the above, we may derive a generalised rule of necessitation for any given notion of relative necessity $\square_{i}$.

Generalised Necessitation If $\phi$ is valid, then $\square_{i} \phi$ is valid.

As a consequence, $\square_{k}\left(\square p \rightarrow \square_{i} p\right)$ is valid whenever $\square_{k}$ and $\square_{i}$ are necessity operators. Using the semantics, one can easily verify that Absolute is valid as a consequence. 
Theorem 3. (Absolute) $\forall p \square_{k}\left(\square p \rightarrow \square_{j} p\right)$ is valid, whenever $\square_{k}$ and $\square_{j}$ are necessity operators.

Relatedly, another advantage of $\mathbf{R N}$ is that there is a sense in which every accessibility relation in a given model generates a notion of relative necessity.

Theorem 4. (Generation) Let $\mathfrak{M}=\langle\mathscr{W}, \mathscr{V}\rangle$ be a model such that $\mathscr{R} \subseteq \mathscr{W}^{2}$. There is a model $\mathfrak{M}_{\mathscr{R}}=\left\langle\mathscr{W}, \mathscr{V}_{\mathscr{R}}\right\rangle$ such that $\left(\mathfrak{M}_{\mathscr{R}}, w\right) \models_{g} \square_{i} \phi$ iff for all u such that $w \mathscr{R} u\left(\mathfrak{M}_{\mathscr{R}}, u\right)=_{g} \phi$.

Proof. For every $w \in \mathscr{W}$, let $\mathscr{R}(w)=\{v: w \mathscr{R} v\}$. Let $\mathscr{V}_{\mathscr{R}}$ be the interpretation function such that for every $w \in \mathscr{W}, p \in \mathscr{V}_{\mathscr{R}}\left(\pi_{i}, w\right)$ iff $\mathscr{R}(w) \subseteq p$, and $\mathscr{V}_{\mathscr{R}}(\xi, w)=\mathscr{V}(\xi, w)$ otherwise. Observe that $w \mathscr{R} u$ iff $u \in \mathscr{R}(w)$ iff $u \in \cap_{\mathscr{W}} \mathscr{V}_{\mathscr{R}}\left(\pi_{i}, w\right)$. Hence $\left(\mathfrak{M}_{\mathscr{R}}, w\right) \models_{g} \square_{i} \phi$ iff for all $u$ such that $w \mathscr{R} u\left(\mathfrak{M}_{\mathscr{R}}, u\right) \models{ }_{g} \phi$.

Both Theorem 4 and Theorem $\mathbf{2}$ are reasonable desiderata on an account of relative necessity. If met, respectively they ensure that to each accessibility relation in a model corresponds an in principle definable notion of relative necessity, and vice-versa. However, notice that SA fails to meet an analogue of Theorem 4, since its notions of relative necessity correspond only to transitive accessibility relations. Similarly, HL2 fails to meet an analogue of Theorem 2. According to HL2, for a given notion of relative $i$-necessity, at $i$-lawless worlds it is $i$-possible that contradictions are true, but on standard model-theoretic treatments of normal modal logics, such notions of relative necessity will not correspond to accessibility relations in models.

\subsubsection{Kripke Necessities}

Complementing the previous section, Theorem 2 and Theorem 4 can be used to establish the neat result that the $\mathbf{R N}$ relative necessities are exactly the Kripke necessities. ${ }^{16}$ To make this claim precise, a number of further definitions are required. First, we define what it is for a modal logic to be the logic of a Kripke frame. The result will then be stated as a correspondence between the logics of relative necessity (according to RN) and the logics of Kripke frames.

Def. 7 The modal propositional language (MPL) is the fragment of $\mathscr{L}$ without the universal quantifier and monadic predicate constants. A modal logic is a set of MPL formulas.

Def. 8 An $M P L$ frame is a pair $\langle\mathscr{W}, \mathscr{R}\rangle$ in which $\mathscr{W}$ is a non-empty set and $\mathscr{R} \subseteq \mathscr{W}^{2}$.

\footnotetext{
${ }^{16}$ Thanks to an anonymous reviewer for conjecturing this theorem and encouraging me to explore it.
} 
Def. 9 An $M P L$ model based on an MPL frame $\mathscr{F}=\langle\mathscr{W}, \mathscr{R}\rangle$ is a triple $\langle\mathscr{W}, \mathscr{R}, \mathscr{V}\rangle$ in which $\mathscr{V}$ is a total function from propositional variables of MPL to subsets of $\mathscr{W}$.

It is helpful to note that for any MPL model $\langle\mathscr{W}, \mathscr{R}, \mathscr{V}\rangle, \mathscr{V}$ is just a variable assignment for an $\mathscr{L}$ model based on the same set of worlds and vice-versa.

Def. 10 ' $(\mathscr{M}, w) \models \phi$ ' is defined for $\phi$ a well-formed MPL formula, $\mathscr{M}=\langle\mathscr{W}, \mathscr{R}, \mathscr{V}\rangle$ an MPL model, and $w \in \mathscr{W}$ recursively in the following manner. In this definition, $\alpha$ is propositional variable, and $\phi$ and $\psi$ are both well-formed MPL formulae.

$$
\begin{aligned}
& (\mathscr{M}, w) \models \alpha \text { iff } w \in \mathscr{V}(\alpha) \\
& (\mathscr{M}, w) \models \neg \phi \text { iff }(\mathscr{M}, w) \vDash \phi \\
& (\mathscr{M}, w) \models \phi \wedge \psi \text { iff }(\mathscr{M}, w) \models \phi \text { and }(\mathscr{M}, w) \models \psi \\
& (\mathscr{M}, w) \models \square \phi \text { iff }(\mathscr{M}, u) \models \phi, \text { for all } u \text { such that } w \mathscr{R} u
\end{aligned}
$$

Def. 11 A formula $\phi$ is valid in an MPL model $\mathscr{M}=\langle\mathscr{W}, \mathscr{R}, \mathscr{V}\rangle$ iff $(\mathscr{M}, w) \models$ $\phi$, for all $w \in \mathscr{W}$. A formula $\phi$ is valid on an MPL frame $\mathscr{F}=\langle\mathscr{W}, \mathscr{R}\rangle$ iff it is valid in all MPL models based on $\mathscr{F}$.

Def. 12 For an MPL frame $\mathscr{F}$, the modal logic of $\mathscr{F}$ is $\Lambda_{\mathscr{F}}=\{\phi \in$ MPL : $\phi$ is valid on $\mathscr{F}$.

Def. 13 For $\phi$ a formula of MPL, $\phi_{i}$ is the formula whose metalinguistic abbreviation is given by the result of substituting each occurrence of $\square$ for an occurrence of $\square_{i}$. (Given the abbreviation conventions for $\square_{i}, \phi_{i}$ will abbreviate a formula of $\mathscr{L}$ but not of MPL.)

Def. 14 For a model $\mathfrak{M}$ of $\mathscr{L}$ (as defined in Def. 2), the modal logic of $\mathfrak{M}$ is $\Lambda_{\mathfrak{M}}^{\mathrm{MPL}}=\left\{\phi \in \mathrm{MPL}: \phi_{i}\right.$ is valid in $\left.\mathfrak{M}\right\}$. (Note the restriction to MPL formulas.)

Def. 15 For a model $\mathfrak{M}=\langle\mathscr{W}, \mathscr{V}\rangle$ of $\mathscr{L}, \mathscr{F}^{i}(\mathfrak{M})$ is the MPL frame $\left\langle\mathscr{W}, \mathscr{R}_{i}\right\rangle$, with $\mathscr{R}_{i}$ defined in terms of $\mathscr{V}$ as in Def. 6.

Def. 16 For an MPL frame $\mathscr{F}=\langle\mathscr{W}, \mathscr{R}\rangle, \mathfrak{M}^{i}(\mathscr{F})$ is the $\mathscr{L}$ model $\mathfrak{M}=\left\langle\mathscr{W}, \mathscr{V}_{\mathscr{R}}\right\rangle$, with $\mathscr{V}_{\mathscr{R}}$ defined in terms of $\mathscr{R}$ as in the proof of Theorem 4.

Theorem 5. (Kripke Necessities) A modal logic is the logic of an $\mathscr{L}$ model iff it is the logic of an MPL frame. 
Proof. We first establish two helpful lemmas.

Lemma 1. For any MPL frame $\mathscr{F}=\langle\mathscr{W}, \mathscr{R}\rangle$ and any MPL model $\mathscr{M}=\langle\mathscr{W}, \mathscr{R}, \mathscr{V}\rangle$ based on $\mathscr{F}$, for all $w \in \mathscr{W},(\mathscr{M}, w) \models \phi$ iff $\left(\mathfrak{M}^{i}(\mathscr{F}), w\right) \models \mathscr{V} \phi_{i}$.

Lemma 1 is established by induction on the complexity of $\phi$. The base case is immediate from the use of $\mathscr{V}$ as the variable assignment over $\mathfrak{M}^{i}(\mathscr{F})$, and the cases for the truth-functional connectives are routine. So assume that for all $w \in \mathscr{W},(\mathscr{M}, w) \models \psi$ iff $\left(\mathfrak{M}^{i}(\mathscr{F}), w\right) \models \mathscr{V} \psi_{i}$. Then reason as follows to complete the proof of Lemma 1.

$(\mathscr{M}, w) \models \square \psi$ iff $(\mathscr{M}, u) \models \psi$ for all $u$ such that $w \mathscr{R} u$

Def. 10

iff $\left.\operatorname{MM}^{i}(\mathscr{F}), u\right) \models \mathscr{V} \psi_{i}$ for all $u$ such that $w \mathscr{R} u$

IH

$$
\text { iff } \left.\operatorname{(M}^{i}(\mathscr{F}), w\right) \models \mathscr{V} \square_{i} \psi_{i}
$$

Theorem 4

Lemma 1 has the following corollary.

Corollary 1. $\Lambda_{\mathscr{F}}=\Lambda_{\mathfrak{M}^{i}(\mathscr{F})}^{\mathrm{MP}}$ for any MPL frame $\mathscr{F}=\langle\mathscr{W}, \mathscr{R}\rangle$.

There is a similar lemma in the other direction.

Lemma 2. For any $\mathscr{L}$ model $\mathfrak{M}=\langle\mathscr{W}, \mathscr{V}\rangle$ and all $w \in \mathscr{W},(\mathfrak{M}, w) \models_{g} \phi_{i}$ iff $(\mathscr{M}, w) \models \phi$, where $\mathscr{M}$ is the MPL model $\left\langle\mathscr{F}^{i}(\mathfrak{M}), g\right\rangle$.

Lemma 2 is also established by induction on the complexity of $\phi$. As before, we only consider the case for necessity claims. So assume that for all $w \in \mathscr{W}$, $(\mathfrak{M}, w) \models_{g} \psi_{i}$ iff $(\mathscr{M}, w) \models \psi$. Then reason as follows to complete the proof of Lemma 2.

$(\mathfrak{M}, w) \models_{g} \square_{i} \psi_{i}$ iff $(\mathfrak{M}, u) \models_{g} \psi_{i}$ for all $u$ such that $w \mathscr{R}_{i} u$

Theorem 2

$$
\begin{aligned}
& \text { iff }(\mathscr{M}, u) \models \psi \text { for all } u \text { such that } w \mathscr{R}_{i} u \\
& \text { iff }(\mathscr{M}, w) \models \square \psi
\end{aligned}
$$

Lemma 2 has the following corollary.

Corollary 2. $\Lambda_{\mathfrak{M}}^{\mathrm{MP}}=\Lambda_{\mathscr{F} i(\mathfrak{M})}$ for any $\mathscr{L}$ model $\mathfrak{M}=\langle\mathscr{W}, \mathscr{V}\rangle$

Theorem 5 then follows from Corollary 1 and Corollary 2.

Theorem 5 shows that $\mathbf{R N}$ meets the initial target of characterising all the relative necessities of interest, the Kripke necessities. It is a significant advantage of $\mathbf{R N}$ that it is able to yield such a result. 


\subsubsection{Lawless Worlds}

Recall that according to HL2 at worlds with no $i$-laws no proposition is $i$-necessary and every proposition is $i$-possible. This was in tension with familiar glosses of $i$ necessity. For example, if physical necessity is simply absolute necessity given the laws of physics, then if there are no laws of physics, there are no propositions to restrict what is physically necessary. Thus, one would expect that physical necessity would collapse into absolute necessity. However, it can be shown that $\mathbf{R N}$ meets this expectation and thus conforms with this familiar gloss of $i$-necessity.

Consider a model $\mathfrak{M}^{1}=\left\langle\mathscr{W}, \mathscr{V}^{1}\right\rangle$ just like $\mathfrak{M}$ in Section 4.2.1 except:

$$
\mathscr{V}^{1}\left(\pi_{i}, w\right)=\emptyset, \text { for all } w \in \mathscr{W}
$$

We want to show that $\left(\mathfrak{M}^{1}, w\right) \models_{g} \square p$ iff $\left(\mathfrak{M}^{1}, w\right) \models_{g} \square_{i} p$ for an arbitrary propositional variable $p$. Of course, the left-to-right direction is immediate from Absolute. But notice that since $\mathscr{V}^{1}\left(\pi_{i}, w\right)=\emptyset, \cap \mathscr{W}^{\mathscr{V}}\left(\pi_{i}, w\right)=\mathscr{W}$. Thus for all $u \in \mathscr{W}$, $w \mathscr{R}_{i} u$. Hence, as intended, when there are no $i$-laws at a given world the $i$-possible worlds from that world are all and only the absolutely possible worlds, which demonstrates the collapse of $i$-necessity and absolute necessity at such $i$-lawless worlds. This is preferable to the verdict of HL2 that even absolutely impossible propositions are $i$-possible at $i$-lawless worlds.

Relatedly, we can note that if the $i$-laws are inconsistent at some world in some model, in the sense that their intersection with respect to the set of all worlds in that model is empty, then at that world everything is $i$-necessary but nothing is $i$ possible. This is another welcome result, for such inconsistent sets of laws and their corresponding relative necessities exhibit a certain degeneracy. To appreciate the result, consider a model $\mathfrak{M}^{\prime}=\left\langle\mathscr{W}, \mathscr{D}, \mathscr{V}^{\prime}\right\rangle$ just like $\mathfrak{M}^{1}$ directly above, except:

$$
\mathscr{V}^{\prime}\left(\pi_{i}, w\right)=\{S, \mathscr{W}-S\}, \text { for some } S \subseteq \mathscr{W}
$$

Speaking informally, it follows that the only proposition (i.e. subset of $\mathscr{W}$ ) which strictly implies all the $i$-laws at $w$ is the impossible proposition, that is $\emptyset$. As a consequence, $w \mathscr{R}_{i} u$ iff $u \in \emptyset$. In other words, there is no $u \in \mathscr{W}$ such that $w \mathscr{R}_{i} u$. Thus, given the derived semantic clause above, it is vacuously true that every $\phi$ is $i$-necessary at $w$ and no $\phi$ is $i$-possible at $w$.

\section{Concluding Remarks}

This article has criticised Hale-and-Leech's [10] account of relative necessity, which itself resulted from their criticisms of the standard account of relative necessity. The paper then proposed a new account of relative necessity which met the criticisms of both Hale \& Leech's account and the standard account.

To close the discussion, it is interesting to note that certain complications arise once various working assumptions are lifted. To begin with, from the outset it was 
made explicit that propositional necessitism was a working assumption of the discussion. If this assumption is lifted, $\mathbf{R N}$ may be an inadequate definition of relative necessity; in such a contingentist setting, the propositional quantifier in $\mathbf{R N}$ will not range over all absolutely possible propositions, depriving $\mathbf{R N}$ of its intended generality. This may come as no surprise: as is typical, non-modal quantificational claims lose their intended generality when simply copied from a necessitist setting and pasted into a contingentist one. When faced with such cases, a natural contingentist reaction has been to offer certain modalised contingentist analogue principles following the lead of Fine [6]. However, as Fine himself and recent commentators have recognised, this modalising strategy is difficult to execute in the case of propositional quantification. ${ }^{17}$ Unfortunately, at the time of writing, such difficulties make it unclear whether an adequate analogue of $\mathbf{R N}$ can be articulated in a contingentist setting. Indeed, clarity on this issue is likely to be gained only if a more general contingentist method of simulating higher-order necessitist discourse can be established.

Finally, it was made explicit that the account relied on a conception of propositions according to which it is absolutely necessary that there is a weakest proposition which strictly implies all $i$-laws. On alternative conceptions of propositions, however, there may be be no such unique proposition. In particular, on some conceptions of propositions they may not form a complete Boolean algebra under the usual truthfunctional operations. For example, in certain cases, there may always be various minimally weak propositions which strictly imply all $i$-laws, but no unique candidate. One would expect that $\mathbf{R N}$ could be adapted to such settings by identifying the $i$-necessity of a proposition with that proposition's being strictly implied by all of the minimally weak propositions which strictly imply all $i$-laws. However, of course, the specific details of the non-Boolean setting matter greatly, so questions regarding how to define relative necessity in such settings are left for future work.

Acknowledgments For helpful discussion and feedback, thanks are due to Arif Ahmed, Robbie Williams, Timothy Williamson, and an audience at the Twelfth Annual Cambridge Graduate Conference on the Philosophy of Mathematics and Logic, at which Arif Ahmed provided comments on the paper. Special thanks are due to James Studd for particularly helpful feedback on earlier drafts, and to two anonymous referees for this journal whose comments greatly improved the paper.

Open Access This article is distributed under the terms of the Creative Commons Attribution 4.0 International License (http://creativecommons.org/licenses/by/4.0/), which permits unrestricted use, distribution, and reproduction in any medium, provided you give appropriate credit to the original author(s) and the source, provide a link to the Creative Commons license, and indicate if changes were made.

\section{References}

1. van Benthem, J. (2010). Modal logic for open minds. CSLI Publications: Stanford.

2. Bacon, A. (2018). The Broadest Necessity. Journal of Philosophical Logic, 47, 733-783.

3. Cresswell, M. (1965). Another basis for S4. Logique and Analyse, 8, 191-195.

\footnotetext{
${ }^{17}$ See in particular Williamson [18, chap. 7] and Goodman \& Fritz [7].
} 
4. Dorr, C. (2010). Iterating definiteness. In Dietz, R., \& Moruzzi, S. (Eds.) Cuts and clouds: vagueness its nature and its logic. Oxford: Oxford University Press.

5. Fine, K. (1970). Propositional quantifiers in modal logic. Theoria, 36, 336-346.

6. Fine, K. (1975). Vagueness, truth, and logic. Synthese, 30, 265-300.

7. Goodman, J., \& Fritz, P. (2017). Counting incompossibles. Mind, 126, 1063-1108.

8. Goranko, V., \& Otto, M. (2006). Model theory of modal logic. In Blackburn, P. et al. (Eds.) Handbook of modal logic. Amsterdam: Elsevier.

9. Hale, B. (1996). Absolute necessities. Philosophical Perspectives, 10, 93-117.

10. Hale, B., \& Leech, J. (2017). Relative necessity reformulated. Journal of Philosophical Logic, 46, 1-26.

11. Humberstone, L. (1981). Relative necessity revisited. Reports on Mathematical Logic, 13, 33-42.

12. Humberstone, L. (2004). Two-dimensional adventures. Philosophical Studies, 118, 17-65.

13. McFetridge, I. (1990). Logical necessity and other essays. London: The Aristotelian Society.

14. Scroggs, S.J. (1951). Extensions of the Lewis system S5. The Journal of Symbolic Logic, 16, 112-120.

15. Smiley, T. (1963). Relative necessity. The Journal of Symbolic Logic, 28, 113-134.

16. Strohminger, M., \& Yli-Vakkuri, J. (2019). Knowledge of objective modality. Philosophical Studies, 176, 1155-1175.

17. Suszko, R. (1971). Identity connective and modality. Studia Logica, 27, 7-39.

18. Williamson, T. (2013). Modal logic as metaphysics. Oxford: Oxford University Press.

19. Williamson, T. (2016). Modal science. Canadian Journal of Philosophy, 46, 453-492.

Publisher's Note Springer Nature remains neutral with regard to jurisdictional claims in published maps and institutional affiliations. 